\title{
Anisotropy of Electroactive Strain in Highly Stretched Polypyrrole Actuators Rachel Pytel $^{\mathrm{ab}}$, Edwin Thomas ${ }^{\mathrm{a}}$, Ian Hunter ${ }^{\mathrm{b}}$
}

\section{Supporting Information}

\section{Polymer preparation:}

Pyrrole (Aldrich 99\%) was vacuum distilled before use. All other materials were used as received. Polypyrrole was electrodeposited at $-40{ }^{\circ} \mathrm{C}$ at a constant current density of $0.5 \mathrm{~A} / \mathrm{m}^{2}$. The deposition solution contained $0.05 \mathrm{M}$ pyrrole, $0.05 \mathrm{M}$ tetraethylammonium hexafluorophosphate (TEAP) and $1 \% \mathrm{vol}$ water in propylene carbonate. Copper foil was used as a counter electrode, and the polypyrrole film was deposited onto a polished glassy carbon working electrode over the course of 18 hours.

After synthesis, large area films $(70 \mathrm{~mm} \times 230 \mathrm{~mm} \times 0.03 \mathrm{~mm})$ were peeled off of the working electrode and trimmed to remove any rough edges. They were then stretched at room temperature, in air, in one of two ways. Small sections $(3 \mathrm{~mm} \times 20 \mathrm{~mm})$ were stretched on a Perkin Elmer DMA 7e Dynamic Mechanical Analyzer in static stress scan mode, at a rate of $1000 \mathrm{kPa} / \mathrm{min}$. This method only produced samples with their long axis parallel to the direction of stretch. Larger sections $(50 \mathrm{~mm} \times 150 \mathrm{~mm})$ were stretched on a Zwick/Roell Z010 Mechanical Tester at a rate of $1 \% / \mathrm{min}$. Samples $(2 \mathrm{~mm} \times 20 \mathrm{~mm})$ were then cut from the larger stretched films, some with their long axis parallel to stretch and some perpendicular to stretch. Samples stretched on the Zwick and then cut with their axis parallel to stretch exhibited the same conductivity as small samples stretched to the same elongation on the Perkin Elmer, so it was assumed that the two methods induced comparable anisotropy for a given elongation. Due to Poisson effects, the width and thickness of all films decreased after stretching, with the highest elongation films having a thickness of only $0.011 \mathrm{~mm}$. Imperfections in the electrodeposited films prevented larger sections from achieving elongations greater than $100 \%$ on the Zwick, while small sections could be stretched over $250 \%$ on the Perkin Elmer DMA.

\section{Measurement:}

Conductivity was measured along the length of each small strip using a standard four wire probe connected to a Keithley 2001 multimeter. WAXS was conducted using a synchrotron radiation source at Brookhaven National Laboratories $(\lambda=1.371 \AA)$. The 2-d wide-angle diffraction patterns are shown in 
Figure 1. The 1-d diffraction curves perpendicular and parallel to stretch for the film stretched $140 \%$ are shown in Figure 3.

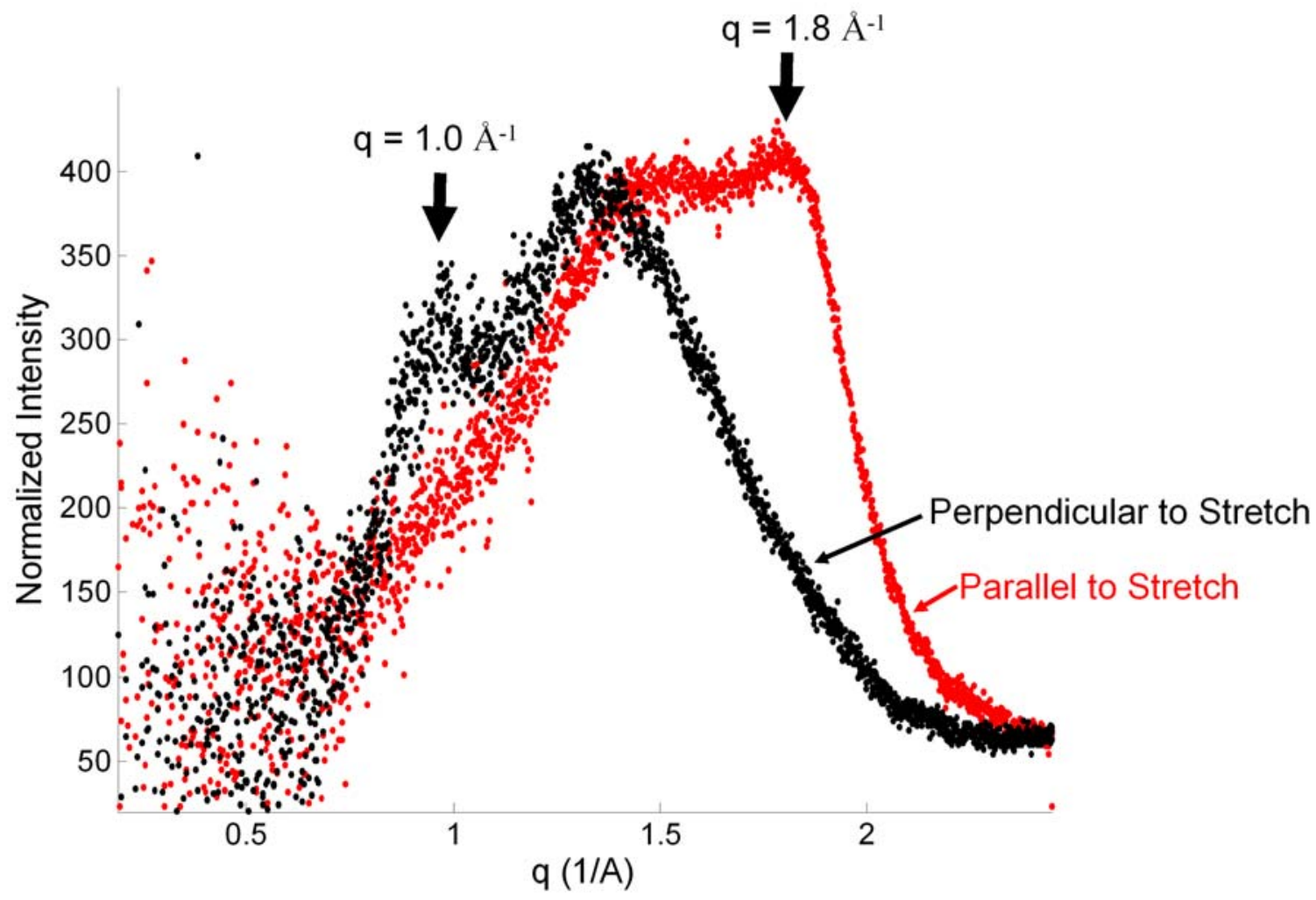

Figure 3: 1-d x-ray diffraction of polypyrrole sample stretched $140 \%$. 2-d wide-angle diffraction data was taken at Brookhaven National Laboratories. To find each 1-d curve, a slice of the 2-d image with width $\Delta \chi=25^{\circ}$ oriented perpendicular or parallel to the stretch axis was integrated using datasqueeze software ${ }^{1}$. Air scattering background was integrated over the same slice, and subtracted from each curve. The peak at $q=1.0 \AA^{-1}$ is found perpendicular to the stretch direction, while the peak at $q=1.8 \AA^{-1}$ is found perpendicular to stretch.

Active strains were measured using an electrochemical dynamic mechanical analyzer developed by Rinderknecht et al. ${ }^{2}$ in isotonic mode. A film was mounted in the electrochemical cell with a solution of 0.05 M TEAP in propylene carbonate, and a constant stress of $0.5 \mathrm{MPa}$ was applied to the film. A sawtooth waveform of $\pm 1.5 \mathrm{~V}$ (vs. $\mathrm{Ag} / \mathrm{Ag}^{+}$) was applied around the open circuit potential, and the resulting length change of the film was recorded. The "active strain" was measured as the difference between the maximum peak strain and the following minimum strain of each voltage cycle, corresponding to the contractile strain in the polymer. 
Alternatively, the rate of polymer creep can be estimated to be linear with time, and subtracted from the electroactive strain data as shown in Figure 4. Using this approach, the magnitude of electroactive strain is the average amplitude over the 5 cycles shown in Figure 4. The unstretched sample of polypyrrole shown in Figure 4 exhibits an active strain of approximately 1.45\%. A film stretched $96 \%$ and tested parallel to stretch presents an active strain of only $0.08 \%$, while a film tested perpendicular to the stretch axis exhibits an active strain of approximately $2.75 \%$. This results in a ratio of perpendicular to parallel active strain $(\varepsilon \perp / \varepsilon \|)_{\text {PPy }}$ of $34: 1$.

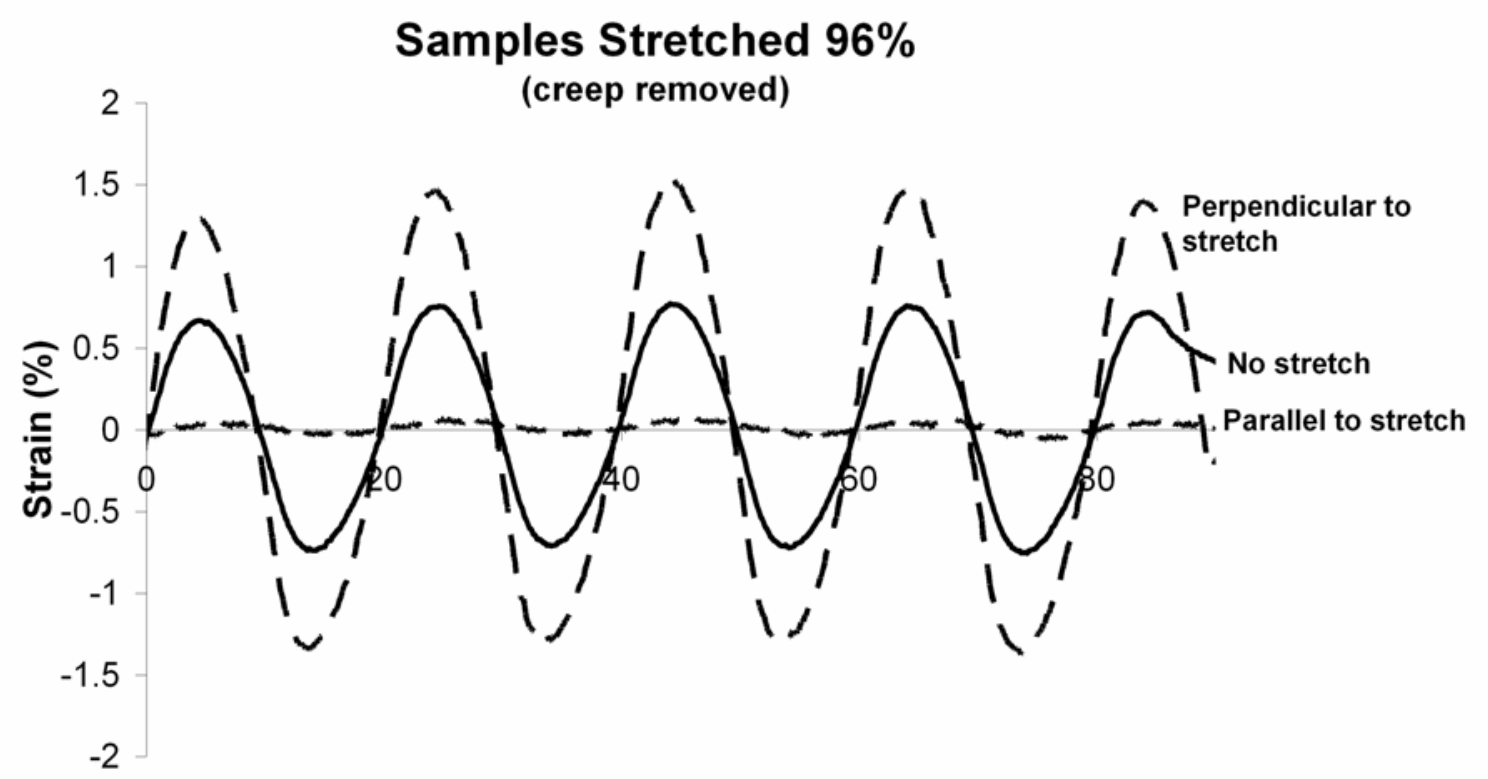

Time (s)

Figure 4: Anisotropic active strain response from stretched films, with linear creep removed. The film cut perpendicular to stretch shows an active strain amplitude of approximately $2.75 \%$, while the film cut parallel to stretch shows a strain amplitude of approximately $0.08 \%$. For these tests a $\pm \mathbf{1 . 5 \mathrm { V }}$ triangular waveform was applied with a frequency of $0.05 \mathrm{~Hz}$.

(1) www.datasqueezesoftware.com

(2) Anquetil, P., Rinderknecht, D., Vandesteeg, N., Madden, J., and Hunter, I.; In Proc. of SPIE $11^{\text {th }}$ Smart Structures and Materials Symp, 2004, 5385, 380. 